\title{
Evaluating the safety and efficacy of argatroban locking solution in the prevention of the dysfunction of haemodialysis central venous catheters: a study protocol for a randomized controlled trial
}

\author{
Yiqin Wang, Chao Liu, Li Zhang, Jijun Li, Lei Zhang, Yong Wang, Hanyu Zhu, Xueying Cao, Di Wu, \\ Jie Wu, Shupeng Lin, Zhe Feng, Guangyan Cai, Xiangmei Chen, Xuefeng Sun \\ Department of Nephrology, Chinese PLA General Hospital, Chinese PLA Institute of Nephrology, State Key Laboratory of Kidney Diseases, \\ National Clinical Research Center for Kidney Diseases, Beijing, China \\ Correspondence to: Xuefeng Sun, MD, PhD. Professor of Nephrology, Department of Nephrology, Chinese PLA General Hospital, Chinese PLA \\ Institute of Nephrology, State Key Laboratory of Kidney Diseases, National Clinical Research Center for Kidney Diseases, 28 Fuxing Road, Beijing, \\ China. Email: xfssun@126.com.
}

Background: Use of anticoagulant as lock solutions is an important method to maintain the function of
haemodialysis (HD) central venous catheters (CVCs), and the common anticoagulants heparin and citrate
are not suitable for some patients. Argatroban can inhibit thrombin directly, has a definite anticoagulant
effect, and is expected to be a new anticoagulant for CVC lock solutions. Methods: A total of $60 \mathrm{HD}$ patients with non-tunnelled or tunnelled CVCs will be randomly assigned to two groups: an argatroban group and a control group. The participants will be given argatroban $0.5 \mathrm{mg} / \mathrm{mL}$ or unfractionated heparin (UFH) $1,000 \mathrm{U} / \mathrm{mL}$ locked post-dialysis instilled into the CVC lumens and followed up for 2 weeks. Data on demographic and general clinical information, laboratory examination, adverse events, adverse reactions and serious adverse events in the two groups will be collected. The differences in coagulation indexes at $30 \mathrm{~min}$ following catheter lock will be compared. The thrombosis rate, infection rate and percentage of catheter-days in the two groups will be observed. The primary outcomes include: efficacy assessments of combined outcome events: (I) rates of cumulative catheter survival in the 2 -week HD session (the standard of catheter survival was catheter mean blood flow $\geq 250 \mathrm{~mL} / \mathrm{min}$ ); (II) rates of cumulative survival free of catheter thrombosis in the 2-week HD session. The second outcomes include: catheter dysfunction, the variation value (seconds) in activated partial thromboplastin time (aPTT) at 30 min following catheter locking and aPTT before next dialysis, catheter-associated bleeding, and catheterassociated infections.

Discussion: At present, there is no clinical study of argatroban as a CVC lock solution. This study will explore the efficacy and safety of the argatroban as locking solution in the prevention of the dysfunction of HD CVCs to provide evidence for further research.

Trial registration: Chinese Clinical Trial Registry, ChiCTR1800017105. Registered 12 July, 2018 (prospectively registered, http://www.chictr.org.cn/showproj.aspx?proj=29054).

Keywords: Haemodialysis catheters; argatroban; unfractionated heparin (UFH)

Submitted Aug 19, 2020. Accepted for publication Dec 24, 2020.

doi: 10.21037/apm-20-1641

View this article at: http://dx.doi.org/10.21037/apm-20-1641 


\section{Introduction}

Haemodialysis (HD) central venous catheters (CVCs) are divided into non-tunnelled catheters and tunnelled catheters. Non-tunnelled catheters are the main vascular access used in continuous renal replacement therapy, extracorporeal membrane oxygenation, extracorporeal blood purification treatment, and urgent HD therapy. Tunnelled catheters are used extensively as permanent vascular access for $\mathrm{HD}$ patients. More than $80 \%$ of $110,000 \mathrm{HD}$ patients in the United States use a CVC every year (1); the use rate of catheters in patients on maintenance HD under 21 years old was much higher than that in other age groups $(52 \%$ vs. 19-21\%) (2). In elderly (over 65 years old) HD patients, even if they receive arteriovenous fistulas or arteriovenous grafts, there are more complications (such as coronary artery or peripheral artery disease); therefore, they have longer CVC dependence times (3). Although the Kidney Disease Outcomes Quality Initiative (K/DOQI) suggests arteriovenous access (arteriovenous fistula or arteriovenous graft) in preference to a CVC in most incident and prevalent HD patients due to the lower infection risk associated with arteriovenous access use, there is inadequate evidence for the KDOQI to make recommendations on the choice of incident vascular access type based on associations with all-cause hospitalizations or mortality (4).

CVC dysfunction is generally due to thrombosis. Radiological investigation of catheters with refractory dysfunction has demonstrated fibrin sheaths in approximately $48 \%$ to $82 \%$ of HD catheters. Use of lock solution is an important method to maintain CVC function. The KDOQI considers it reasonable to use citrate or heparin as a CVC locking solution (4). However, the use of heparin can result in allergy and heparin-induced thrombocytopenia (HIT) and even cause potentially life-threatening complications of thrombosis (5-8). The prevalence of HIT in HD patients was 3.9-17.9\% (9). A high-concentration heparin locking solution increases the risk of bleeding $(10,11)$. The results of risk analysis showed that the risk of cardiovascular events could be induced by a citrate lock solution, especially with a high-concentration citrate solution, in the case of foreseeable operational errors (12). Therefore, there are patients in whom heparin and citrate are not applicable.

Argatroban has been used in the clinic for more than 20 years, and its anticoagulant efficacy and safety have been widely verified. Argatroban has been approved in the United States, the European Union and Japan for the prevention or treatment of thrombosis in patients with
HIT and anticoagulation in patients with HIT undergoing percutaneous coronary intervention (PCI) (13).

Argatroban can reversibly bind directly with the thrombin active site to inhibit thrombin-mediated fibrinogen transformation, inhibit thrombin-induced platelet activation through the protease-activated receptor-1 (PAR-1) receptor (14), prevent thrombin activatable fibrinolysis inhibitor (TAFI) activation and promote endogenous fibrinolysis by inhibiting the thrombin-thrombomodulin complex (13). Therefore, argatroban not only can prevent thrombosis in CVCs but also can treat thrombosis in CVCs. The half-life of argatroban is 39-51 min (15). Among endstage renal disease (ESRD) patients, HD increased the clearance rate of argatroban by approximately $20 \%$ and reduced the half-life to $35 \pm 6 \mathrm{~min}$ (16). If a small amount of argatroban leaks into the patient's systemic circulation after locking, it can be quickly metabolized, and the risk of adverse reactions such as bleeding can be further reduced. Argatroban is mainly metabolized by the liver, and the effect on renal function can be ignored $(14,17,18)$.

Based on the pharmacological and pharmacokinetic characteristics of argatroban, we believe that argatroban is expected to be a new anticoagulant for CVC locking solution. At present, there is no clinical study of argatroban as a CVC lock solution. This study will explore the efficacy and safety of the argatroban as locking solution in the prevention of the dysfunction of HD CVCs to provide evidence for further research. We present the following article in accordance with the SPIRIT reporting checklist (available at http://dx.doi.org/10.21037/apm-20-1641) (19).

\section{Methods}

\section{Objectives}

An exploratory evaluation of the efficacy and safety of argatroban in the prevention of CVC thrombosis to provide basic data for making argatroban lock a potential alternative to heparin as a catheter lock.

\section{Trial design}

This study is a single-centre, prospective, open-label randomized controlled trial (RCT). According to the order of patients enrolled in the trial, the random number generated by Excel (Microsoft) was used for randomization. The study is designed as a primary and exploratory study. It is hypothesized that the effectively and safely of argatroban as CVC lock solution not inferior to heparin sodium 
$(1,000 \mathrm{U} / \mathrm{mL})$. The study protocol was designed by members of the Executive Committee (composed of the Department of Nephrology of the Chinese People's Liberation Army General Hospital). The protocol is being implemented by the Department of Nephrology of the Chinese People's Liberation Army General Hospital. The study will conduct in accordance with the Declaration of Helsinki (as revised in 2013). This trial received ethical approval from the Medical Ethics Committee of the Chinese People's Liberation Army General Hospital on 31 May, 2018 (IRB No. S2018-055-01), and was registered with Chinese Clinical Trial Registry (ChiCTR1800017105, http://www.chictr.org.cn/showproj.aspx?proj=29054) on 12 July 2018. Patients who agree to participate and sign the informed consent will be involved. Approximately 60 ESRD patients with HD CVC will be enrolled in the group and followed up for 2 weeks (Figure 1).

\section{Eligibility, exclusion and exit criteria}

The inclusion criteria are as follows: (I) adult patients (age $>18$ years); (II) a diagnosis of acute kidney injury or chronic renal failure and receiving HD; (III) vascular pathway was non-tunnelled catheters or tunnelled catheters; (IV) HD mode: 3 times/week, HD session is $4 \mathrm{~h}$; and (V) agreement to participate and the provision of signed informed consent.

The exclusion criteria are as follows: (I) systemic anticoagulation; (II) a history of heparin allergy or HIT; (III) argatroban allergy; (IV) CVC in use has a history of infection or dysfunction; (V) systemic or local infection before enrolment; (VI) malignant tumour; (VII) pregnancy or lactation; and (VIII) participation in another clinical investigation.

The exit criteria are as follows: (I) participant withdraws informed consent; (II) participant unable to tolerate the adverse reaction; (III) the decision of kidney transplantation; (IV) dialysis mode changes (stop HD for more than 1 week or change to peritoneal dialysis); (V) drug allergy that cannot be relieved by antiallergic drugs; (VI) according to the investigator's judgement, the patient should not continue the trial due to adverse events (AEs) or abnormal laboratory test values; (VII) participants experience serious diseases that require the cessation of the trial treatment; (VIII) trial termination is the choice in the best interests of the patient; (IX) serious breach of protocol, including participants failing to take medications according to protocol or taking some drugs that have a significant impact on the primary and secondary outcomes; (X) serious adverse events (SAEs), including hospitalization (initial or prolonged), disability or permanent damage, lifethreatening SAEs, death, congenital anomaly/birth defects and other serious events (medical events).

\section{Primary outcome}

\section{Efficacy assessments}

Efficacy assessments of combined outcome events including: (I) rates of cumulative catheter survival in the 2-week HD session (the standard of catheter survival was catheter mean blood flow $\geq 250 \mathrm{~mL} / \mathrm{min}$ ); and (II) rates of cumulative survival free of catheter thrombosis in the 2-week HD session

\section{Secondary outcome}

Catheter dysfunction: (I) insufficient blood flow rate during dialysis (a peak blood flow $<200 \mathrm{~mL} / \mathrm{min}$ for $30 \mathrm{~min}$, or the mean blood flow $<250 \mathrm{~mL} / \mathrm{min}$ during two consecutive dialysis sessions); (II) the formation of blood clots observed when the blood from the catheter is drawn back to the syringe before dialysis (poor patency or no blood flow); (III) ultrasonography revealed thrombosis of CVC.

\section{Safety assessments}

(I) The variation value (seconds) in activated partial thromboplastin time (aPTT) at $30 \mathrm{~min}$ following catheter locking and aPTT before next dialysis (2nd HD day) (the reference range of variation value is 1.5-2.5 times of aPTT of the patient before the first dialysis after randomization).

(II) The incidence of bleeding or bleeding aggravating events, and the severity of bleeding was defined according to International Society on Thrombosis and Haemostasis (ISTH) (normal range: adult male $<4$ points, adult female $<6$ points).

(III) The incidence of catheter-related infection, defined by one of the following events: any purulent secretion is present at the exit site of the catheter, any tunnelled catheter-related infection are present and any symptoms of catheter-related blood infection (such as fever and shivering are present).

\section{Interventions}

\section{Argatroban group}

At the end of dialysis, the patients were randomly given 


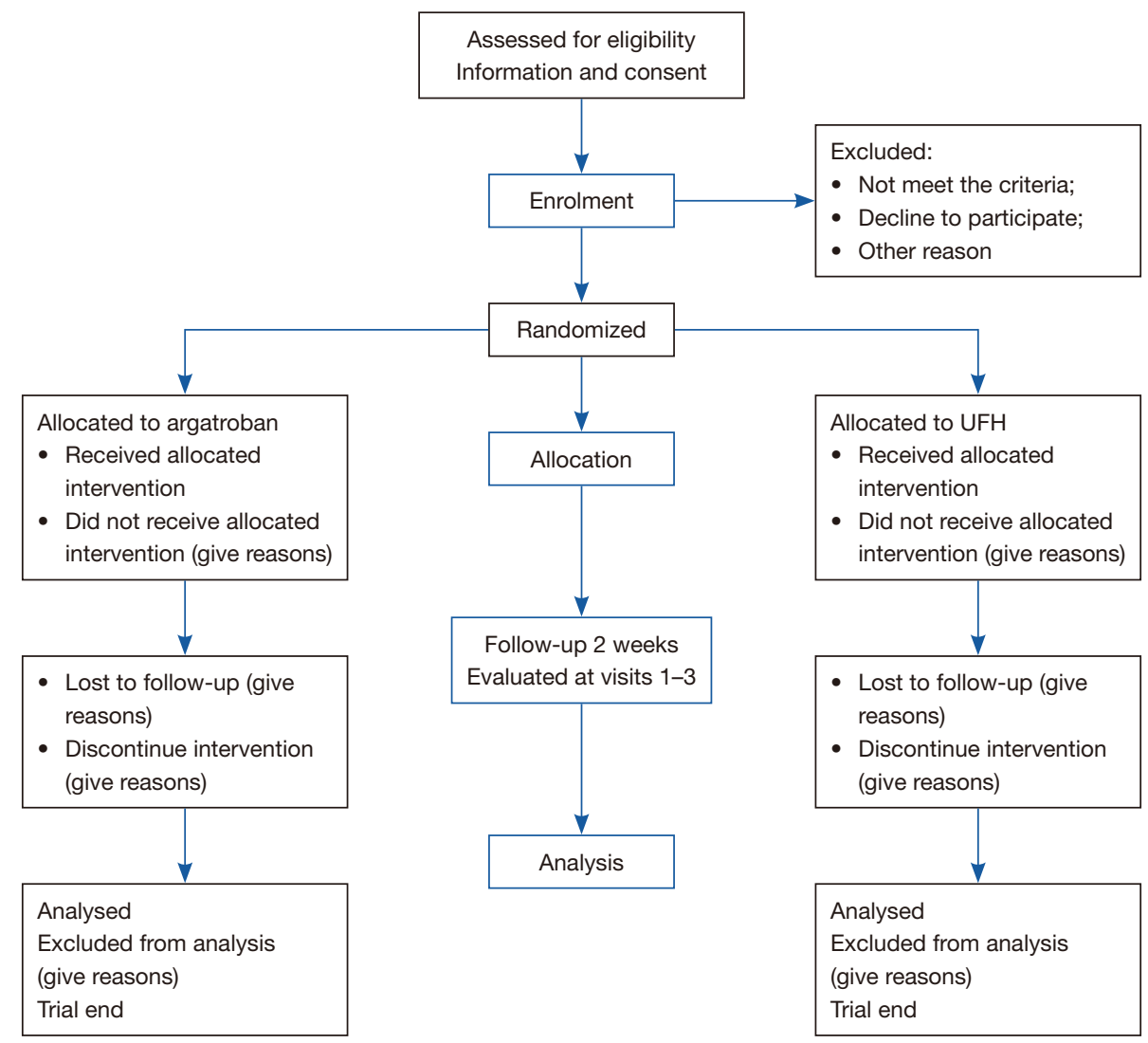

Figure 1 Trial flowchart.

argatroban injection $0.5 \mathrm{mg} / \mathrm{mL} \times 4 \mathrm{~mL}$ equal volume instilled into the CVC lumens and followed up for 2 weeks. The frequency of treatment was 3 times a week.

\section{Unfractionated heparin (UFH) group}

At the end of dialysis, the patients were randomly given heparin sodium injection $1,000 \mathrm{U} / \mathrm{mL} \times 4 \mathrm{~mL}$ equal volume instilled into the CVC lumens and followed up for 2 weeks. The frequency of treatment was 3 times a week.

\section{Administration protocol}

For the argatroban group, the nurses will aspirate $4 \mathrm{~mL}$ of argatroban $(20 \mathrm{~mL} / 10 \mathrm{mg}$, produced by TIPR Pharmaceutical Responsible Co., Ltd, Tianjin, China) into a 5 -mL syringe and produce $4 \mathrm{~mL}$ of argatroban $0.5 \mathrm{mg} / \mathrm{mL}$ as the lock agent per patient per dialysis run $(2 \mathrm{~mL}$ for both venous and arterial lumen).

For the heparin group, the nurses will aspirate $1 \mathrm{~mL}$ of
6,250 U/mL heparin (produced by SPH No.1 Biochemical \& Pharmaceutical Co., Ltd.) into a $10-\mathrm{mL}$ syringe and add $5 \mathrm{~mL}$ of sterile normal saline to produce approximately $6 \mathrm{~mL}$ of heparin $1,000 \mathrm{U} / \mathrm{mL}$ as per the current standard of care ( $2 \mathrm{~mL}$ per catheter lumen). The locking agent will remain in the catheter lumen until the next HD run, and at the beginning of the next run, the solution will be withdrawn and discarded. During the trial, the doses of heparin and Argatroban were fixed and did not change.

\section{Catheter care protocols}

The routine care of all dialysis catheters will be performed by nurses wearing masks and gloves to lock the catheter and change dressing at the outlet of the catheter. During each dialysis session, the catheter outlet will be checked. If there are any signs of potential infection (redness, exudation or tenderness at the exit), a sensitivity test will be performed and, if necessary, swabs will be sent for culture. 
Table 1 Items and procedures of the trial at baseline and each follow-up

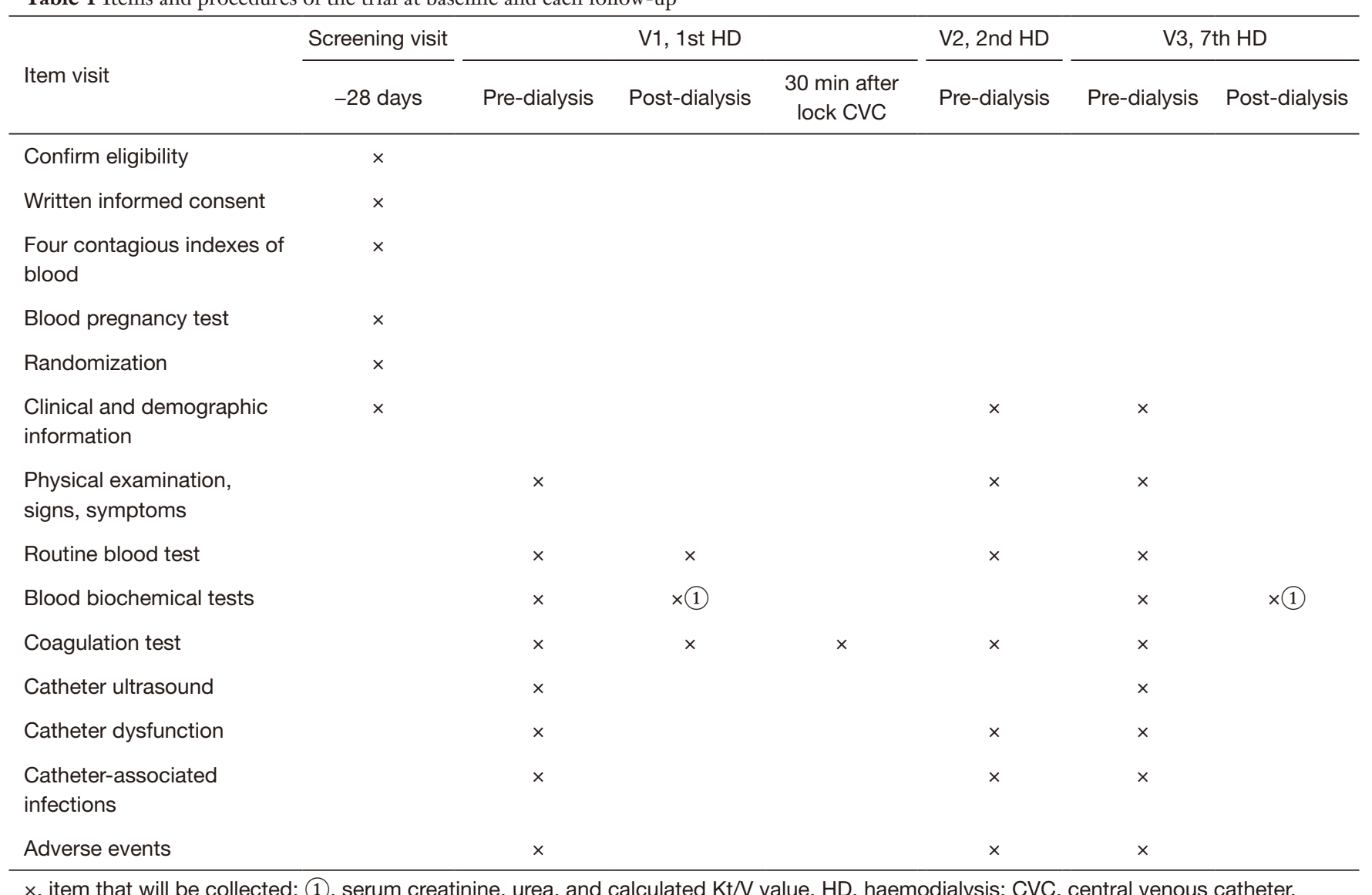

\section{Screening stage}

Visit 0 (screening period): (I) perform screening and assessment and obtain informed consent. (II) Collect clinical and demographic information, including age, sex, primary disease and comorbidity. (III) Measure height and weight of patients; collect vital signs (blood pressure, pulse, body temperature); determine CVC creation date and previous vascular access; record medical history and concomitant medications; and perform a detailed laboratory examination, including blood routine parameters, blood biochemistry (serum albumin, blood lipid, serum creatinine, urea), coagulation state (aPTT), pregnancy test (for childbearing age) and CVC ultrasonography (Table 1). Blood samples $(10 \mathrm{~mL})$ will be collected during screening period.

\section{Treatment stage}

The treatment stage includes visit 1 (week 1 , first dialysis: 1st HD), visit 2 (week 1, second dialysis: 2 nd HD), and visit 3 (week 3, first dialysis: 7th HD), and the duration of treatment is 2 weeks.

At the end of the first dialysis after randomization, the participant will begin to receive either argatroban or heparin locking solution in the catheter. They will be interviewed at V1, V2 and V3 to collect the general clinical data and the aPTT data, to evaluate the function of the catheter and to determine the presence of catheter-related infection and bleeding events. Blood pressure, pulse and body temperature will be measured at each visit, and the combined medication and AEs will be recorded. Blood samples $(20 \mathrm{~mL})$ will be collected during V1, V2 and V3 (Table 1). In clinical trials, if the platelet count is more than $50 \%$ lower than the baseline, the HIT cannot be excluded, and serum samples should be frozen in case of a required post-hoc analysis (20).

\section{Concomitant medication (intervention)}

The participants are forbidden to take anticoagulants, 
antiplatelet drugs and fibrinolytic drugs intravenously or orally; the drugs used to treat hypertension, anaemia, chronic kidney disease-mineral and bone disorder (CKD$\mathrm{MBD}$ ) and other complications are to remain unchanged during the observation period.

\section{Sample size}

The purpose of this study was to explore the efficacy and safety of argatroban in the prevention of CVC thrombosis. Since there are almost no previous studies on argatroban as lock solution, and this study is an exploratory experiment and does not belong to the category of evidence-based medicine, it is impossible to calculate the effective sample size according to the method of evidence-based medicine. Therefore, the sample size was set at 30 cases in each group, a total of 60 cases.

\section{Randomization and blinding method}

Randomization by 1:1 distribution can reduce the deviation caused by the expectation of patients or doctors, and the computer-generated random number method will be used for random grouping. This study is an open-label study, and there is no blinding method. Patients who meet the inclusion criteria and provide informed consent will be randomly divided into two groups.

\section{Data collection and management}

Table 1 gives an overview of assessment tests and the times of data collection. An overview of registration information is shown in Table 2. For each patient randomly assigned to a group, baseline and follow-up data will be saved regardless of their final completion of the trial. Patients participating in the trial will be given priority treatment and free examination. Clinical investigators will collect the data and fill in the case report form (CRF). To ensure complete first-hand information, we will also design specific medical records for the clinical trial. Scientific researchers will write the medical records while following up patients to ensure that the data records are completed in a timely, accurate and correct manner. The inspectors are responsible for checking the consistency of all data sources, paper case reports and electronic databases. The original medical records and informed consent will be archived in the participating centre and kept for at least 5 years after the end of the clinical trial. During the whole experiment, the CRF information will be input into the EpiData database designed by the experiment for management. At the end of the experiment, two people will verify the CRF data input.

\section{Statistical methods}

\section{Analysis data set}

(I) Full analysis set (FAS): randomized patients will be included and analysed according to the principle of intention to treat (ITT).

(II) Per protocol set (PPS): All patients who complete follow-up and do not seriously violate the protocol will be included.

(III) Safety set (SS): The actual data of at least one treatment with safety index records will be included.

We will use SPSS 25.0 for statistical analysis. Participants' demographic, clinical, and laboratory data will be described using the mean [standard deviation (SD)] or the median (range), depending on the underlying distribution, for continuous variables, or the frequency (percentage) for categorical variables. Continuous variables will be compared using the $t$-test or the Wilcoxon rank sum test where appropriate. Categorical variables will be compared using the chi-squared test. A $\mathrm{P}$ value of 0.05 for two-sided univariate tests will be considered statistically significant. Kaplan-Meier survival analysis will be performed on an ITT basis for rates of cumulative catheter survival and AE-free survival and the rate of catheterrelated infection. The Cox proportional hazards regression model will be used to determine predictors of time to next catheter dysfunction. Multiple interpolation is used to deal with the missing data, and the missing value is incorporated into multiple regression as a group of data. Statistical significance was defined as $\mathrm{P}<0.05$.

\section{The main analysis contents}

(I) Comparability analysis: demographic data and general clinical data will be compared to assess the comparability of the two groups.

(II) Compliance analysis: compare the situation of participants taking drugs on schedule and at the proper dose in two groups, as well as banned drugs in unused schedule.

(III) Effectiveness analysis: the differences in coagulation indexes at $30 \mathrm{~min}$ following catheter lock will be compared. The thrombosis rate, infection rate and percentage of catheter-days in the two groups will be observed. 
Table 2 Trial registration data set

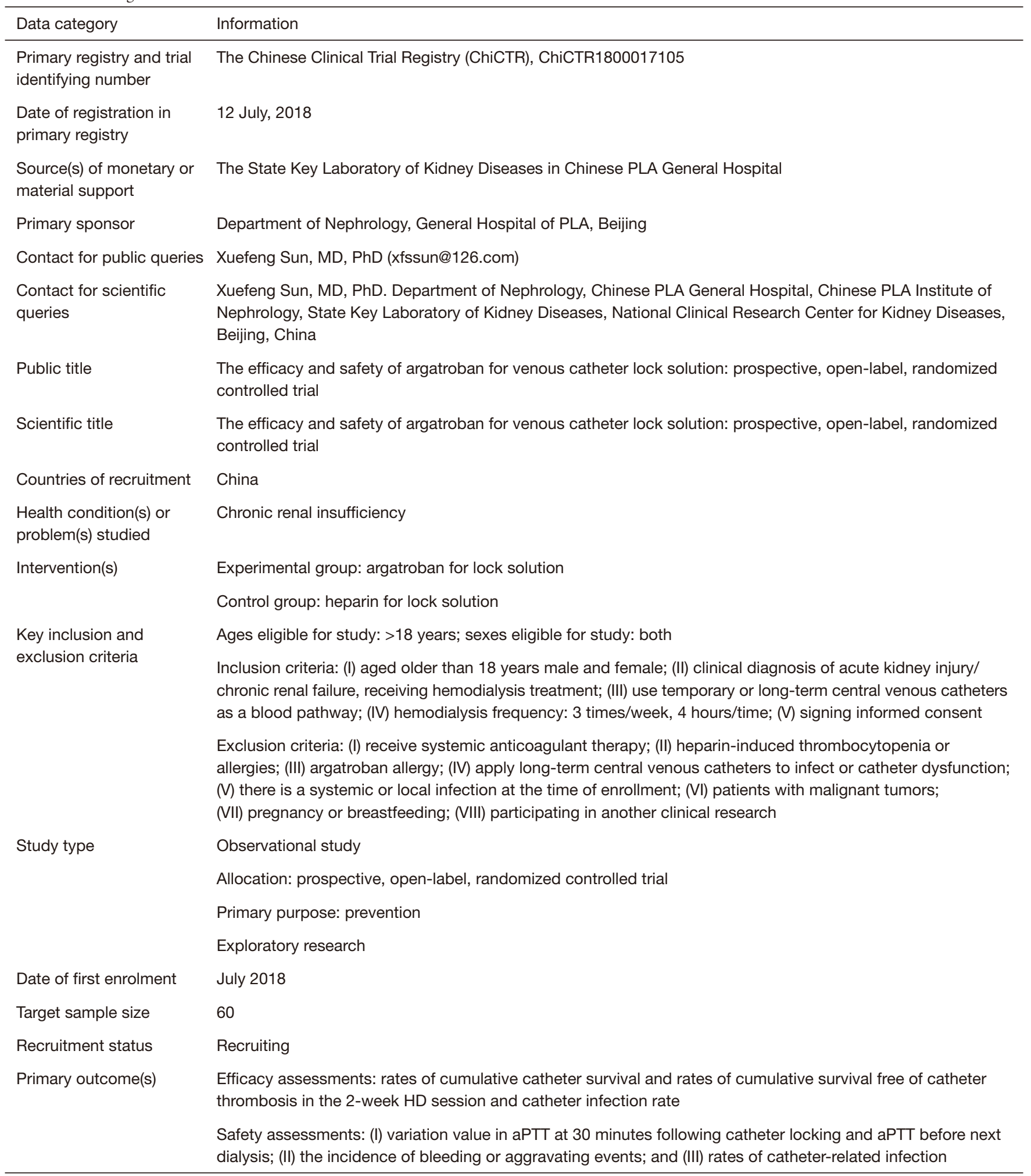

Table 2 (continued) 
Table 2 (continued)

\begin{tabular}{|c|c|}
\hline Data category & Information \\
\hline \multirow{2}{*}{ Key secondary outcomes } & $\begin{array}{l}\text { Inclusion criteria: (I) aged older than } 18 \text { years male and female; (II) clinical diagnosis of acute kidney injury/ } \\
\text { chronic renal failure, receiving hemodialysis treatment; (III) use temporary or long-term central venous catheters } \\
\text { as a blood pathway; (IV) hemodialysis frequency: } 3 \text { times/week, } 4 \text { hours/time; (V) signing informed consent }\end{array}$ \\
\hline & $\begin{array}{l}\text { Exclusion criteria: (I) receive systemic anticoagulant therapy; (II) heparin-induced thrombocytopenia or } \\
\text { allergies; (III) argatroban allergy; (IV) apply long-term central venous catheters to infect or catheter dysfunction; } \\
\text { (V) there is a systemic or local infection at the time of enrollment; (VI) patients with malignant tumors; (VII) } \\
\text { pregnancy or breastfeeding; (VIII) participating in another clinical researcher }\end{array}$ \\
\hline \multirow[t]{3}{*}{ Study type } & Observational study \\
\hline & Allocation: prospective, open-label, randomized controlled trial \\
\hline & Primary purpose: prevention \\
\hline Target sample size & 60 \\
\hline Recruitment status & Recruiting \\
\hline Primary outcome(s) & $\begin{array}{l}\text { Efficacy assessments: (1) rates of cumulative catheter survival and rates of cumulative survival free of catheter } \\
\text { thrombosis in the 2-week HD session and catheter infection rate } \\
\text { Safety assessments: (1) variation value in aPTT at } 30 \text { minutes following catheter locking and aPTT before next } \\
\text { dialysis; (2) the incidence of bleeding or aggravating events; and (3) rates of catheter-related infection }\end{array}$ \\
\hline Key secondary outcomes & Catheter dysfunction, catheter-associated infections, catheter-associated bleeding \\
\hline
\end{tabular}

$\mathrm{HD}$, haemodialysis; aPTT, activated partial thromboplastin time.

(IV) Security analysis: the distribution of AEs, adverse reactions and SAEs in the two groups will be analysed. The patient's name, start date, end date, severity, and the correlation and influence of the trial medicine, along with the measures taken and the outcome, will be described in detail in the list.

\section{Data monitoring}

As a data monitoring committee (DMC), the clinical research management centre of PLA General Hospital will be responsible for coordinating and supervising test activities, maintaining and filing test documents, purchasing and distributing test materials, implementing statistical planning and analysis, and providing results analysis.

\section{Harms}

Potential AEs include bleeding, gastrointestinal reactions, allergic reactions, hypotension, and fever. During the clinical trial, all AEs (including screening period and treatment period) during the drug treatment will be recorded in the CRF. Any SAEs related to or unrelated to the investigational drug during the trial session (from the time of obtaining informed consent until 30 days after the last administration or the last visit, whichever is the latest) must be reported to the ethics committee of PLA General Hospital within 24 hours after the investigator first knows about them. At the same time, researchers must fill in the records of SAEs, including the time, severity and duration of the SAE, as well as measures and results.

\section{Auditing and inspection}

During or after the study, the PLA General Hospital regulatory agencies will conduct inspection to ensure proper research and data processing procedures are carried out as required. 


\section{Ethics and dissemination}

Prior to the start of the trial, the protocol, informed consent and other relevant documents were submitted to the medical ethics committee [Human Research Ethics Committee (HREC)] of PLA General Hospital for written approval. Any subsequent substantive revision of the approved document shall be resubmitted to the abovementioned organization for approval. The investigator is responsible for explaining the purpose, method, benefits and potential risks to each subject and obtaining informed consent. All patient data will be saved according to the assigned serial number, and the private information will be protected. No one will get the data until the analysis is complete. We plan to publish the results of the experiment in appropriate journals and share the results in academic conferences. The report will be prepared by the executive committee, and our final report will follow the guidelines of the comprehensive report test standard (Consort) (21).

\section{Discussion}

Thrombus and stenosis are important factors of CVC dysfunction. Tunnelled HD catheter dysfunction generally occurs due to thrombosis, and the category referred to as a "fibrin sheath" is the most common culprit (22). Radiological investigation of catheters with refractory dysfunction has demonstrated fibrin sheaths in approximately $48 \%$ to $82 \%$ of HD catheters (23-25). Therefore, the application of anticoagulant lock solution is the routine maintenance method of CVCs.

Heparin is the traditional and most commonly used CVC lock solution. UFH not only inhibits the activity of clotting factors Xa, IXa, XIa and XIIa but also the activity of thrombin directly. However, heparin does not promote fibrinolysis and has no therapeutic effect on formed thrombus. Moreover, since there is no blood in CVCs after the injection of heparin, there is no antithrombin.

The anticoagulant effect of UFH can only be attributed to the reflux infiltration of antithrombin in the bloodstream of the patients after locking the catheter; however, in this process, it must be accompanied by the reflux of thrombin, forming fibrin and gradually forming a thrombus.

Therefore, in theory, heparin lock agents cannot completely prevent catheter thrombosis. Many studies have also shown that a heparin lock can only partially reduce the occurrence of catheter dysfunction (26-28). In addition, in vitro experiments confirmed that heparin stimulated the formation of Staphylococcus aureus biofilms, which increased with increasing heparin concentration and stimulation time (29).

Citrate-chelated calcium ions not only prevent the conversion of prothrombin to thrombin but also inhibit the conversion of fibrinogen to fibrin, which has a good anticoagulant effect. In vitro studies have confirmed that more than $0.5 \%$ citrate can effectively inhibit the proliferation and biofilm formation of $S$. aureus and Staphylococcus epidermidis (30). Therefore, citrate not only can inhibit CVC thrombosis but also can prevent CVC-related infection. In a meta-analysis of 21 studies (4,832 patients, 318,769 catheter-days), the incidence of catheter-related bloodstream infections (CRBSIs) and exit-site infections (ESIs) was significantly lower in the citrate-based regimen than in the heparin-based regimen (31). However, similar to heparin, citrate has no fibrinolytic effect and has no therapeutic effect on formed thrombi. In addition, a citrate lock has a risk of inducing cardiovascular events (12). In vitro, citric acid at concentrations greater than $12 \%$ could significantly promote protein agglutination dominated by albumin. In vivo studies demonstrated that protein agglutination in tubes after locking catheters with $46.7 \%$ and $20 \%$ citrate (32) and $43 \%$ citrate locking solution had the risk of inducing pulmonary embolism (33).

As mentioned, argatroban inhibits thrombin activity and platelet activation and promotes endogenous fibrinolysis $(13,14)$. Moreover, argatroban effectively inhibited thrombin binding to old fibrin clots or thrombin binding to fibrin clots that had been treated with streptokinase or alteplase. Argatroban inhibited approximately $85 \%$ of thrombin activity in these old fibrin clots, and the saturated concentration of argatroban inhibited thrombin in clots treated with streptokinase or alteplase by approximately $70 \%$ (34). Therefore, argatroban not only can prevent the process of thrombosis in CVC but also can treat formed thrombi in CVCs. In terms of pharmacology, argatroban as a lock solution has more advantages than heparin and citric acid.

The most significant adverse effect of argatroban was haemorrhagic complications, but it was not common (24). Argatroban is non-immunogenic and generally does not cause allergic reactions (25). Its most common adverse reaction is mild gastrointestinal disorder. Due to the short half-life of argatroban and its limited capacity to leak into the catheter (16), it can be predicted that the adverse reactions of argatroban are very limited and that the safety is good. 
In conclusion, argatroban is more advantageous than heparin or citric acid in terms of pharmacological action, and it may become a new CVC locking solution. However, there is a lack of clinical research on argatroban as a locking agent. The results of this study will provide evidence for the efficacy and safety of argatroban as a CVC locking solution and provide basic data for further multicentre, large-sample, randomized control studies. This study has good clinical value, and the results are expected.

\section{Acknowledgments}

Funding: This work was supported by the National Natural Science Foundation of China (81870463), National Key R\&D Program of China (2018YFC0114503) and by funds from the State Key Laboratory of Kidney Diseases in Chinese PLA General Hospital (KF201807).

\section{Footnote}

Reporting Checklist: The authors have completed the SPIRIT reporting checklist. Available at http://dx.doi.org/10.21037/ apm-20-1641

Conflicts of Interest: All authors have completed the ICMJE uniform disclosure form (available at http://dx.doi. org/10.21037/apm-20-1641). The authors have no conflicts of interest to declare.

Ethical Statement: The authors are accountable for all aspects of the work in ensuring that questions related to the accuracy or integrity of any part of the work are appropriately investigated and resolved. The study will conduct in accordance with the Declaration of Helsinki (as revised in 2013). This trial received ethical approval from the Medical Ethics Committee of the Chinese People's Liberation Army General Hospital on 31 May 2018 (IRB no. S2018-055-01), and was registered with Chinese Clinical Trial Registry (ChiCTR1800017105, http://www. chictr.org.cn/showproj.aspx? proj=29054) on 12 July 2018. Patients who agree to participate and sign the informed consent will be involved.

Open Access Statement: This is an Open Access article distributed in accordance with the Creative Commons Attribution-NonCommercial-NoDerivs 4.0 International License (CC BY-NC-ND 4.0), which permits the noncommercial replication and distribution of the article with the strict proviso that no changes or edits are made and the original work is properly cited (including links to both the formal publication through the relevant DOI and the license). See: https://creativecommons.org/licenses/by-nc-nd/4.0/.

\section{References}

1. Saran R, Li Y, Robinson B, et al. US Renal Data System 2015 Annual Data Report: Epidemiology of Kidney Disease in the United States. Am J Kidney Dis 2016;67:Svii, S1-305.

2. US Renal Data System 2019 Annual Data Report: Epidemiology of Kidney Disease in the United States. Am J Kidney Dis 2019. [Epub ahead of print]. doi: 10.1053/ j.ajkd.2019.09.002.

3. Kim HY, Bae EH, Ma SK, et al. Association between initial vascular access and survival in hemodialysis according to age. Korean J Intern Med 2019;34:867-76.

4. Lok CE, Huber TS, Lee T, et al. KDOQI Clinical Practice Guideline for Vascular Access: 2019 Update. American Journal of Kidney Diseases 2020;75:S1-S164.

5. Chong BH. Heparin-induced thrombocytopenia. J Thromb Haemost 2003;1:1471-8.

6. Reilly RF. The pathophysiology of immune-mediated heparin-induced thrombocytopenia. Semin Dial 2003;16:54-60.

7. Hirsh J, Heddle N, Kelton JG. Treatment of heparininduced thrombocytopenia: a critical review. Arch Intern Med 2004;164:361-9.

8. O'Shea SI, Ortel TL, Kovalik EC. Alternative methods of anticoagulation for dialysis-dependent patients with heparin-induced thrombocytopenia. Semin Dial 2003;16:61-7.

9. Yamamoto S, Koide M, Matsuo M, et al. Heparin-induced thrombocytopenia in hemodialysis patients. Am J Kidney Dis 1996;28:82-5.

10. Karaaslan H, Peyronnet P, Benevent D, et al. Risk of heparin lock-related bleeding when using indwelling venous catheter in haemodialysis. Nephrol Dial Transplant 2001;16:2072-4.

11. Hryszko T, Brzosko S, Mysliwiec M. Low concentration of heparin used for permanent catheters canal locking is effective and diminishes the risk of bleeding. Int Urol Nephrol 2013;45:825-9.

12. Polaschegg HD, Sodemann K. Risks related to catheter locking solutions containing concentrated citrate. Nephrol Dial Transplant 2003;18:2688-90.

13. Jeske WP, Fareed J, Hoppensteadt DA, et al. Pharmacology 
of argatroban. Expert Rev Hematol 2010;3:527-39.

14. Swan SK, Hursting MJ. The pharmacokinetics and pharmacodynamics of argatroban: effects of age, gender, and hepatic or renal dysfunction. Pharmacotherapy 2000;20:318-29.

15. Cruz-Gonzalez I, Lopez-Jimenez R, Perez-Rivera A, et al. Pharmacokinetic evaluation of argatroban for the treatment of acute coronary syndrome. Expert Opin Drug Metab Toxicol 2012;8:1483-93.

16. Murray PT, Reddy BV, Grossman EJ, et al. A prospective comparison of three argatroban treatment regimens during hemodialysis in end-stage renal disease. Kidney Int 2004;66:2446-53.

17. Guzzi LM, McCollum DA, Hursting MJ. Effect of renal function on argatroban therapy in heparininduced thrombocytopenia. J Thromb Thrombolysis 2006;22:169-76.

18. Hursting MJ, Murray PT. Argatroban anticoagulation in renal dysfunction: a literature analysis. Nephron Clin Pract 2008;109:c80-94.

19. Spirit 2013. Evid Based Dent 2013;14:120.

20. Harenberg J, Jorg I, Fenyvesi T, et al. Treatment of patients with a history of heparin-induced thrombocytopenia and anti-lepirudin antibodies with argatroban. J Thromb Thrombolysis 2005;19:65-9.

21. Piaggio G, Elbourne DR, Pocock SJ, et al. Reporting of noninferiority and equivalence randomized trials: extension of the CONSORT 2010 statement. JAMA 2012;308:2594-604.

22. Beathard GA. Dysfunction of new catheters by old fibrin sheaths. Semin Dial 2004;17:243-4.

23. Gray RJ, Levitin A, Buck D, et al. Percutaneous fibrin sheath stripping versus transcatheter urokinase infusion for malfunctioning well-positioned tunneled central venous dialysis catheters: a prospective, randomized trial. J Vasc Interv Radiol 2000;11:1121-9.

Cite this article as: Wang Y, Liu C, Zhang L, Li J, Zhang L, Wang Y, Zhu H, Cao X, Wu D, Wu J, Lin S, Feng Z, Cai G, Chen X, Sun X. Evaluating the safety and efficacy of argatroban locking solution in the prevention of the dysfunction of haemodialysis central venous catheters: a study protocol for a randomized controlled trial. Ann Palliat Med 2021;10(2):2260-2270. doi: 10.21037/apm-20-1641
24. Savader SJ, Haikal LC, Ehrman KO, et al. Hemodialysis catheter-associated fibrin sheaths: treatment with a lowdose rt-PA infusion. J Vasc Interv Radiol 2000;11:1131-6.

25. Hoshal VL Jr, Ause RG, Hoskins PA. Fibrin sleeve formation on indwelling subclavian central venous catheters. Arch Surg 1971;102:353-8.

26. Thomson PC, Morris ST, Mactier RA. The effect of heparinized catheter lock solutions on systemic anticoagulation in hemodialysis patients. Clin Nephrol 2011;75:212-7.

27. Ivan DM, Smith T, Allon M. Does the heparin lock concentration affect hemodialysis catheter patency? Clin J Am Soc Nephrol 2010;5:1458-62.

28. Han X, Yang X, Huang B, et al. Low-dose versus highdose heparin locks for hemodialysis catheters: a systematic review and meta-analysis. Clin Nephrol 2016;86:1-8.

29. Shanks RM, Donegan NP, Graber ML, et al. Heparin stimulates Staphylococcus aureus biofilm formation. Infect Immun 2005;73:4596-606.

30. Malo J, Jolicoeur C, Theriault F, et al. Comparison between standard heparin and tinzaparin for haemodialysis catheter lock. ASAIO J 2010;56:42-7.

31. Chen CH, Chen YM, Yang Y, et al. Re-Evaluating the Protective Effect of Hemodialysis Catheter Locking Solutions in Hemodialysis Patients. J Clin Med 2019;8:412.

32. Schilcher G, Scharnagl H, Horina JH, et al. Trisodium citrate induced protein precipitation in haemodialysis catheters might cause pulmonary embolism. Nephrol Dial Transplant 2012;27:2953-7.

33. Cardinal P, Allan J, Pham B, et al. The effect of sodium citrate in arterial catheters on acid-base and electrolyte measurements. Crit Care Med 2000;28:1388-92.

34. Hantgan RR, Jerome WG, Hursting MJ. No effect of clot age or thrombolysis on argatroban's inhibition of thrombin. Blood 1998;92:2064-74. 